\title{
Effect of pulse chirp on distributed Brillouin fiber sensing
}

\author{
Carlos A. Galindez ${ }^{*}$, Luc Thévenaz. \\ Laboratory of Metrology and Photonics, EPFL Swiss Federal Institute of Technology, \\ STI NAM Station 11, CH-1015 Lausanne, Switzerland.
}

\begin{abstract}
The influence of the frequency chirp inherent to the generation of a pump pulse by a Mach-Zehnder intensity modulator is presented in the context of a distributed Brillouin sensor configuration. This frequency chirp depends on the pulse shape and its impact is related to the relative importance of the rising and falling edges with respect to the total pulse width. This results in a biased evaluation of the Brillouin frequency in regions where an abrupt change of this Brillouin frequency is observed.
\end{abstract}

Keywords: Brillouin fiber sensor, Chirp factor, Mach-Zehnder Interferometer

\section{INTRODUCTION}

A common and efficient configuration for Brillouin distributed fiber sensing uses the so-called pump and probe technique $^{1}$, in which a pump pulse interacts with a $\mathrm{CW}$ probe giving rise to a local coupling when the frequency difference between pump and probe precisely matches the Brillouin Stokes shift. To fully preserve the coherence and the frequency stability of the pump light this optical wave is pulsed from the light emitted by a stable CW laser using an external intensity modulator. In most situations this modulator is a Mach-Zehnder electro-optic guided-wave modulator, since it may offer minimized spurious modulation effects, large bandwidth and excellent extinction ratio. Moreover it may even generate from the same laser the probe frequency-shifted CW signals ${ }^{2}$.

If the push-pull phase shifts induced in each arm of the Mach-Zehnder are identical, a pure intensity modulation is obtained. But in a real modulator a slight misbalance is always observed that does not impact on the intensity modulation depth, but results in a superimposed unwanted phase modulation. The chirp is defined by a parameter $\alpha$ that relates the phase change to the normalized light intensity change ${ }^{3}$.

The modulator phase chirp potentially affects the response of a Brillouin distributed sensor by inducing a frequency modulation through the pump pulse. This way the instantaneous frequency carried by the pulse turns also modulated. The effect is shown in Fig. 1: during the rising and falling edges of the pulse the phase is continuously sweeping, giving rise to an instantaneous frequency shift. The optical frequency at the front and trailing edges of the optical pulses is therefore shifted with respect to the central flat part of the pulse.

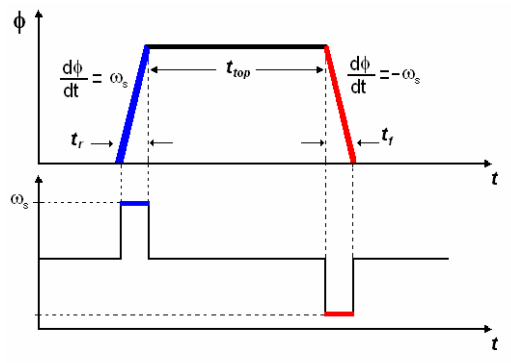

(a)

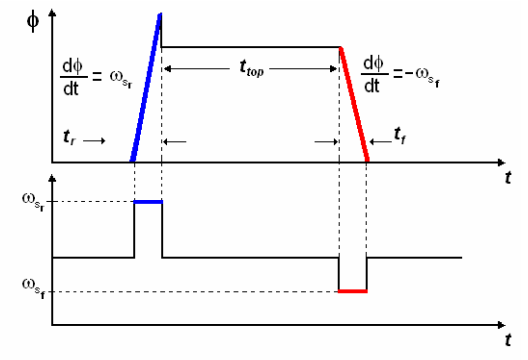

(b)

Fig. 1. Phase change (top) induced by an electro-optic modulator during the generation of the pulse and corresponding instantaneous frequency (bottom): a) trapezoidal pulse shape. b) Irregular pulse with overshoot.

\footnotetext{
* On leave from Photonics Engineering Group, ETSII y de Teleco, University of Cantabria, Av. Castros s/n, 39005, Santander, Spain; galindezca@unican.es; phone +34 942 200877; fax +34 942200877
} 
The effect will be particularly sensitive when an abrupt step-like change of the Brillouin frequency is experienced (hot spot, sharp strain, etc...). When the front part of the pulse is entering the section of fiber showing an abrupt change of the Brillouin frequency, this part of the pulse will sense this section with a shifted frequency and will thus provide a biased shifted Brillouin gain spectrum. The biasing will be similar in magnitude but showing an opposite frequency shift when the pulse is exiting the section. The frequency shift will be larger for a fast phase sweeping, thus for fast rising and falling pulse edges. But the interaction length over which the frequency is shifted will be shorter in this case and the relative importance of the biased pulse sections will be reduced. Reversely long leading and trailing edges will give moderate frequency shifts, but longer relative interaction lengths and will eventually impact more severely on the measurement. The extreme case is represented by the triangular pulse in which no section of the pump pulse carries the nominal optical frequency.

\section{SIMULATION AND EXPERIMENTAL DEMONSTRATION}

An optical fiber shows a natural and well defined Brillouin frequency. This frequency is linearly shifted by temperature or strain of the surrounding medium; when there is a change in one of these environmental quantities in a definite fiber section, this creates two zones described by their proper Brillouin frequency (Fig. 2). In a distributed Brillouin sensor this frequency is evaluated by sweeping the optical frequency of the probe wave. This sweeping is normally realized using one of the sidebands generated in an electro-optic modulator ${ }^{2}$. If this sideband matches the local Brillouin frequency of the medium, the probe wave is amplified and the Brillouin frequency is determined by the frequency giving the maximum amplification. The pump wave is pulsed to make the Brillouin interaction local along the fiber.

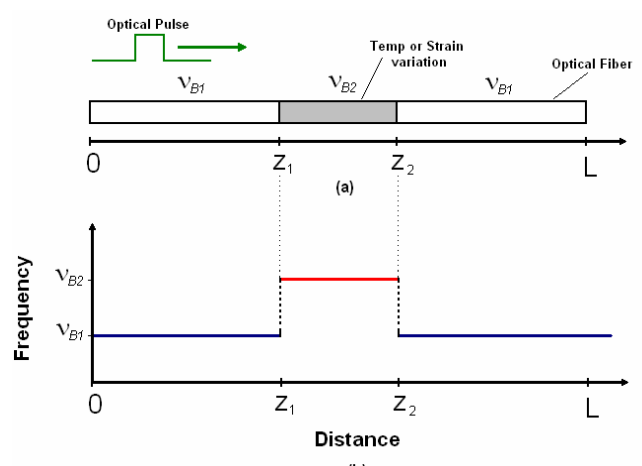

(b)

Fig. 2. a) Experimental configuration to test the effect of the modulator chirp; $v_{B l}$ represents the natural Brillouin frequency of fiber and is the Brillouin frequency of a singular section (e.g. the temperature or the strain on the fiber is changed). b) Corresponding distribution of the Brillouin shift.

Let assume a pump light pulse such as shown in Fig. 1. When the leading edge of the pulsed light, which has a positive frequency chirp, enters the singular section of the fiber, the Brillouin frequency apparently shifts to the blue. The same analysis can be applied to the trailing part of the pulsed light that sees a red shift in the estimation of the local Brillouin frequency. This effect can be totally misinterpreted as a shifted value of the instantaneous Brillouin frequency with a non varying pulsed pump frequency. The relative importance of the interaction length for the shifted and unshifted sections of the pulse will of course scale the importance of the biasing on the Brillouin gain spectrum. The coupled equations that describe the spatial evolution of pump and probe amplitudes can be used to simulate the chirp influence on the Brillouin frequency along the optical fiber.

To perform simulations faithfully reproducing experimental conditions, the test fiber was considered as homogeneous except along a fiber segment that shows a $25 \mathrm{MHz}$ offset of the Brillouin frequency with respect to the homogeneous section $\left(v_{B 2}=v_{B 1}+25 \mathrm{MHz}\right)$, to replicate the scheme shown in Fig. 2 . The pulse width is set to 10ns; since this value offers relatively important rising and falling times. The electrical pulse applied to the electrodes was assumed to present typical features observed in a real situation. For instance a pulse overshoot has a substantial impact through the chirping effect. Topping overshoots are particularly harmful since they give rise to important phase changes in the section of the pulse where the intensity is large. This section of course essentially contributes to the optical Brillouin interaction.

Simulations were carried out for non regular phase variations on trapezoidal and irregular-trapezoidal pulses in order to introduce more realistic approximations. Result for an irregular pulse showing an overshoot like in Fig. 1b is shown in 


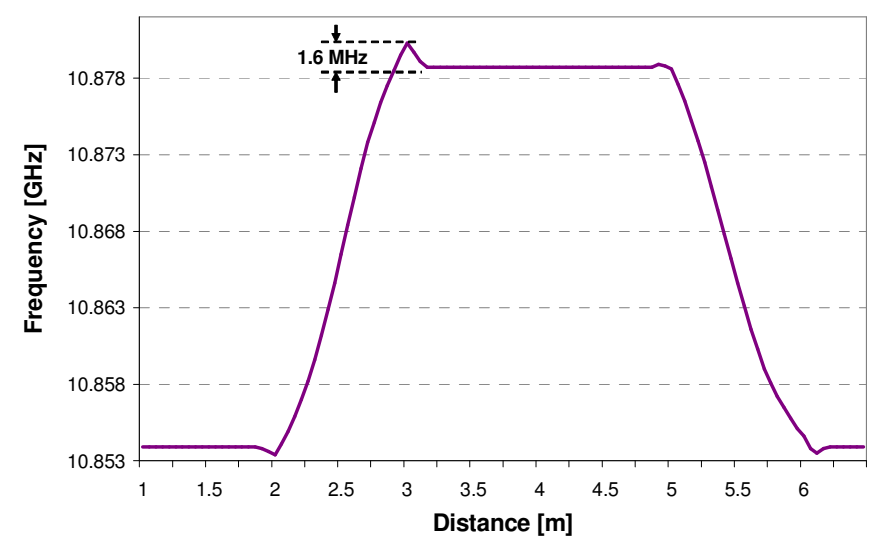

Fig. 3. Calculated Brillouin frequency as a function of position for an irregular trapezoidal pulse, simulated for a $0.1 \mathrm{rad} / \mathrm{s}$ chirp value. Pulse characteristics are: $10 \mathrm{~ns}$ width, $1.5 \mathrm{~ns}$ rise/fall time, overshoot is $15 \%$ over the pulse top.

Fig. 3. Here, it is important to point out the extra frequency shift at the transition between the 2 fiber sections. This extra shift of $1.6 \mathrm{MHz}$ is obtained for a chirp parameter of $0.1 \mathrm{rad} / \mathrm{s}$, which is typical for commercial Mach-Zehnder electrooptic modulators. The effect of chirp is not limited to the transition region but offsets the measurement of the Brillouin frequency all over the fiber length. This offset is a consequence of the asymmetric trapezoidal pulse shape that manifests through a different frequency shift for leading and trailing edges, as shown in Fig. 1b. This asymmetry results in distorted skewed measured Brillouin gain spectra and a biased evaluation of their central frequency.

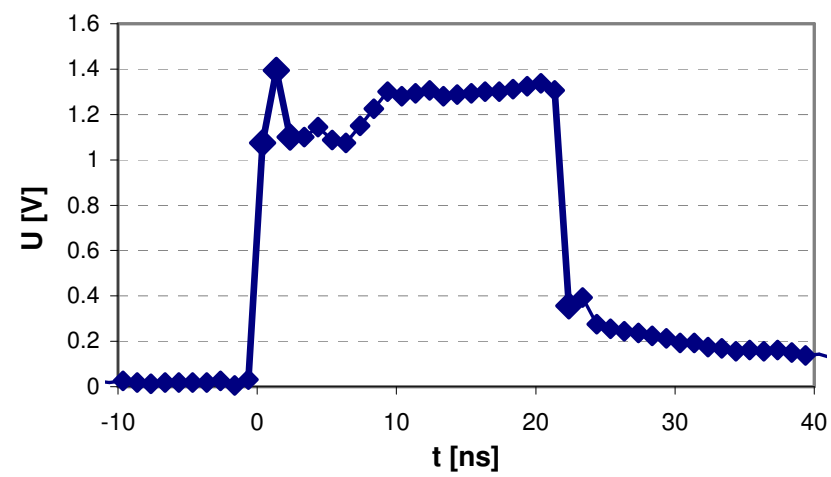

Fig. 4. Real electrical pulse used to simulate and experimentally test the effect of chirp, presenting an overshoot on the leading edge. Typical rise and fall times are $1 \mathrm{~ns}$.

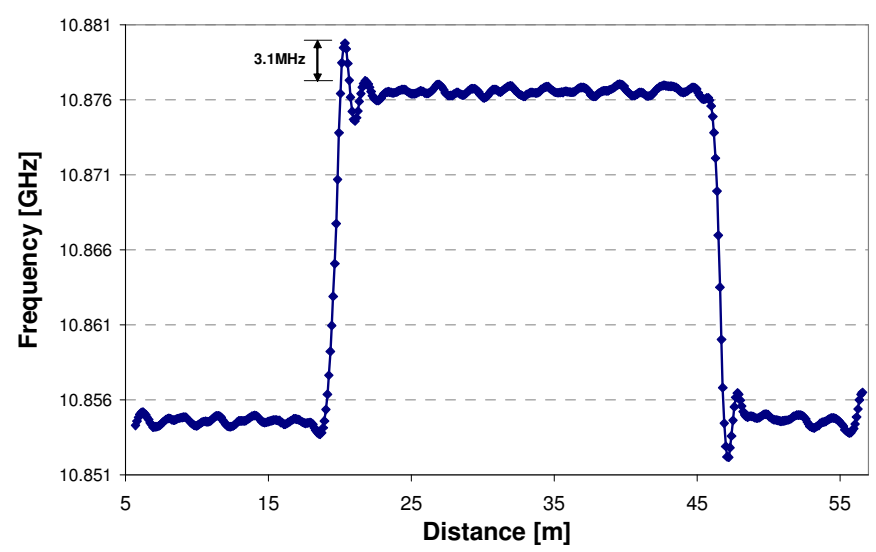

Fig. 5. Experimental demonstration of the effect of chirp, illustrated by the measurement of the Brillouin frequency as a function of position in the vicinity of a $25 \mathrm{~m}$ fiber segment showing a step change of the Brillouin frequency (see configuration shown in Fig. 2). The electrical pulse applied on the modulator electrodes is shown in Fig. 4. 
The effect of the modulator chirp was also proved experimentally by creating a step change of the Brillouin frequency by splicing two optical fibers with close values of the Brillouin frequency, as sketched in Fig. 2. The Brillouin frequencies of the 2 fibers were $12.7974 \mathrm{GHz}$ and $12.8434 \mathrm{GHz}$, respectively. The real electrical pulse applied on the electrodes is shown in Fig. 3 characterized by an important, but realistic overshoot. The measured values of the Brillouin frequency using a standard setup for distributed Brillouin analysis ${ }^{2}$, with pulse width of $10 \mathrm{~ns}$ and typical shape as in Fig. 4 , is shown in Fig. 5. Spurious peaks in the measured Brillouin frequency in the regions of transition between the fibers are clearly observed, leading to a $>3 \mathrm{MHz}$ misevaluation of the Brillouin frequency.

\section{DISCUSION AND CONCLUSIONS}

In this paper we demonstrate through simulations and experimental tests that a standard chirp of $0.1 \mathrm{rad} / \mathrm{s}$ in a modulator used for forming pump pulses in a Brillouin sensor can affect the evaluation of the Brillouin frequency in a nonnegligible manner, with deviations far exceeding the potential accuracy of such sensors. The effect is particularly important when the pulse shows a pronounced overshoot on the leading edge.

Chirp biasing can be minimized by paying attention to the following points when designing the setup:

1. Selection of a modulator with a reduced chirp parameter $\alpha$.

2. Pulse shaping with sharp rising and falling edges. The drawback would be a broader pulse spectrum.

3. Overshoots in the electrical driving pulse are highly detrimental and must be totally suppressed.

On the other hand the effect of chirp can be efficiently cancelled out by using a sequence of electrical pulses with equal amplitude but reverse polarity. With the DC bias on the modulator properly set for a full extinction, positive or negative electrical pulses will give identical light intensity modulation, but the frequency offset resulting from the phase chirp will be reversed for inversed polarity. Since the acquisition is averaged over many pulses the effect of chirp will also average to a null value using such an electrical pulse sequence with alternatively switched polarity.

\section{ACKNOWLEDGMENTS}

We acknowledge the support from the Spanish Government under the research projects TEC2004-05936-CO2-02 and TEC2007-67987-C02-01.

\section{REFERENCES}

1. T.Horiguchi, T.Kurashima, M.Tateda, "A technique to measure distributed strain in optical fibers", Photonics Tech. Lett., 2, p. 352, 1990.

2. M. Niklès, L. Thévenaz, P.A. Robert. "Simple distributed fiber sensor based on Brillouin gain spectrum analysis". Opt. Lett. Vol 21, p 758-760. 1996.

3. F. Koyama and K. Iga, "Frequency chirping in external modulators," J. Lightwave Technol., vol. 6, pp. 87-93, 1988. 